\title{
Tagungsbericht
}

Daphné Çetta* und Joachim Griesbaum

\section{Informationskompetenz und Demokratie (IDE): Bürger, Suchverfahren und Analyse-Algorithmen in der politischen Meinungsbildung}

\author{
Eröffnungstagung des Projekts am 18. und 19. Oktober 2019 in Hildesheim
}

https://doi.org/10.1515/iwp-2019-2064

\section{Das Projekt}

An der von Professor Dr. Joachim Griesbaum und Professor Dr. Thomas Mandl (Institut für Informationswissenschaft) sowie Professorin Dr. phil. Elke Montanari (Institut für Sprache und Literatur) der Stiftung Universität Hildesheim veranstalteten Tagung nahmen insgesamt 89 Personen unterschiedlicher Fachrichtungen teil. Vertreten waren sowohl die Informationswissenschaft, das Bibliothekswesen, die Lehramtsausbildung als auch Studentinnen und Studenten sowie interessierte Bürgerinnen und Bürger, die miteinander in Kontakt traten und sich zu Themen der Informationskompetenz austauschten.

Die Eröffnungstagung wurde im Rahmen des Projekts „Informationskompetenz und Demokratie (IDE): Bürger, Suchverfahren und Analyse-Algorithmen in der politischen Meinungsbildung“ durchgeführt. Das Projekt wird durch das Niedersächsische Ministerium für Wissenschaft und Kultur im Rahmen der Ausschreibung „Zukunftsdiskurse“ aus Mitteln des Niedersächsischen Vorab gefördert.

Ralf Knackstedt, Sprecher des Zentrums für den digitalen Wandel der Universität Hildesheim, begrüßte die Anwesenden. Er führte aus, dass Informationskompetenz als Fachbegriff zwar trocken klinge, jedoch jeden etwas anginge und im Alltag sehr präsent sei.

\footnotetext{
*Kontaktperson: Daphné Çetta, Stiftung Universität Hildesheim, Institut für Informationswissenschaft und Sprachtechnologie, Universitätsplatz 1, 31141 Hildesheim,

E-Mail: cetta@uni-hildesheim.de

Prof. Dr. Joachim Griesbaum, Stiftung Universität Hildesheim, Institut für Informationswissenschaft und Sprachtechnologie, Universitätsplatz 1, 31141 Hildesheim, E-Mail: griesbau@uni-hildesheim.de
}

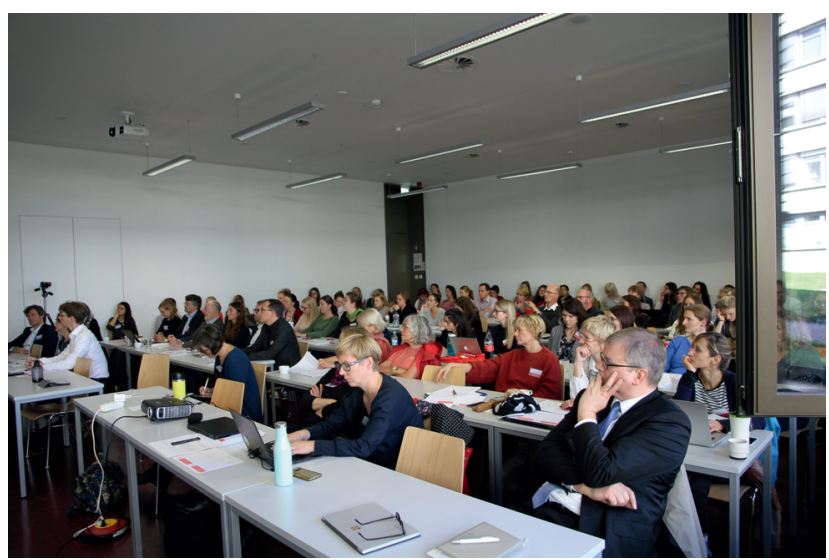

Abbildung 1: Die Auftaktveranstaltung war gut besucht. (Foto: Uni Hildesheim)

\section{Eröffnungsvortrag}

Den Eröffnungsvortrag zur Tagung hielt Lisa Merten (Leibniz-Instituts für Medienforschung | Hans-Bredow-Institut). Sie thematisierte Praktiken, Prinzipien und Pathologien der informationsorientierten Mediennutzung. Dabei zeigte sie auf, dass das Internet zwar einen kontinuierlichen Anstieg als Nachrichtenquelle verzeichne, Social Media allerdings nicht bzw. nur in einem verschwindend geringen Maß als Nachrichtenquelle genutzt würden. Des Weiteren erläuterte sie verschiedene Stufen der Meinungsbildung. Neue Phänomene stellen dabei bspw. Fake News Filterblasen, Social Bots und Echokammern dar. Intermediäre, wie Social Networks, Foto- und Videoplattformen, Messaging-Dienste und Suchmaschinen würden unterschiedlich starke Wirkungen auf die Phasen im Meinungsbildungsprozess haben und in diesem Zuge informations- und medienbezogene Kompetenzen von Nutzern erfordern. 


\section{Informationsraum Internet}

Joachim Griesbaum (Universität Hildesheim) thematisierte das Internet als utopischen und dystopischen Informationsraum. Das Internet weise einerseits vielfältige Wissensbestände auf und ermögliche einen ubiquitären Informationszugriff, andererseits stelle es auch einen schwer zu durchdringenden Informationsdschungel dar. Das Internet als Informationsraum beschrieb er als komplex, intransparent und interessengesteuert. Gründe dafür seien bspw. Regulierungstendenzen, die Macht einiger weniger dominanter Akteure auf wichtigen Informationsmärkten wie Google oder Facebook, die auf Gewinnerzielung fokussiert seien, das Verbreiten von Fake-News sowie unsichtbare und intransparente Algorithmen. Des Weiteren ging Griesbaum auf das Suchverhalten der Nutzer ein. Diese würden zumeist dazu neigen, den Aufwand bei der Suche gering $\mathrm{zu}$ halten. Schließlich wurde argumentiert, dass neben dem Wissen zur Informationsumwelt und einer Motivation sich informationskompetent zu verhalten auch eine kritische, rationale Sicht auf die Realität und die Bereitschaft neues, fremdes Wissen anzunehmen, für gelebte Informationskompetenz erforderlich seien.

\section{Förderung von Informationskompetenz}

Thomas Mandl (Universität Hildesheim) ging in seinem Vortrag auf die folgenden vier Handlungsfelder ein, die im Kontext der Informationskompetenz von Bedeutung seien: Suche, Geschäftsmodelle, Datennutzung und künstlichen Intelligenz. Dabei seien Suchfunktionen für eine Vielzahl von Themen nutzbar, jedoch könne diskutiert werden, ob auch bei allen Anfragen Ergebnisse erscheinen sollten (bspw. bei Gesundheitsthemen). Zudem bestehe nur wenig Kenntnis über die Funktionsweise von Suchmaschinen. Das Suchverhalten vieler Nutzerinnen und Nutzer beschränke sich auf wenige Tools, wenige Suchbegriffe und wenige Ergebnisse. Des Weiteren wurde die Datennutzung thematisiert. In diesem Zusammenhang ging es um von den Suchenden online generierte Daten, die für Dritte interessanten seien (Bsp.: Verkauf von biometrischen Daten in Australien). Abschließend wurde auf die Entwicklungen im Bereich künstlicher Intelligenz eingegangen. Auch Texte könnten von Computern innerhalb weniger Sekunden generiert werden, daher könne man sich nicht mehr völlig sicher sein, ob ein vorliegender Text von einem Menschen oder einer Maschine erzeugt wurde. Insgesamt müsse herausgearbeitet werden, dass neue Dienste zu einem veränderten und komplexeren Begriff von Informationskompetenz führten.

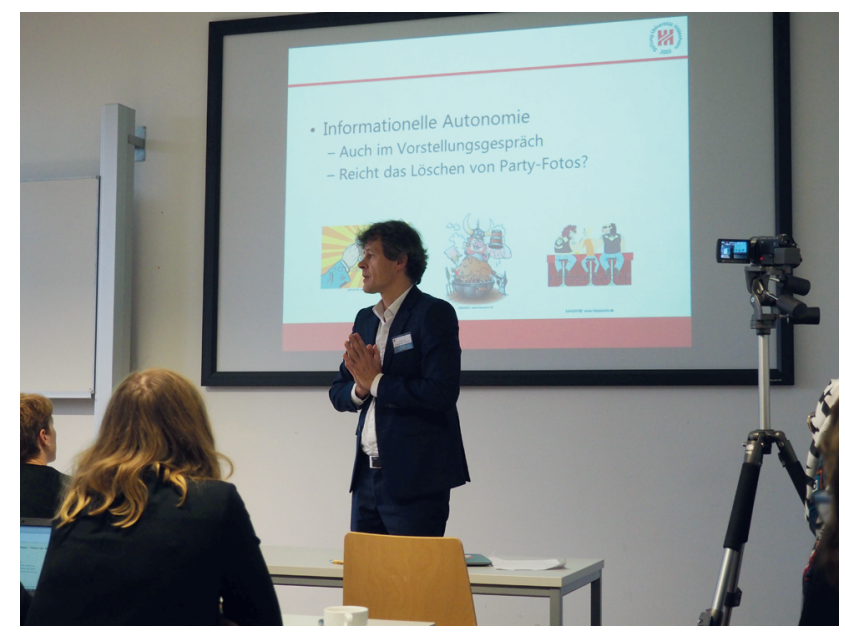

Abbildung 2: Prof. Dr. Thomas Mandl bei seinem Vortrag. (Foto: Uni Hildesheim)

Elke Montanari und Judith Scharf (Universität Hildesheim) behandelten in ihrem Vortrag die Förderung der Informationskompetenz bei Kindern. Zunächst ging Montanari auf die curricularen Vorschriften ein, welche die klassischen Medien, wie z. B. Bücher, Gedichte etc. behandeln. Heute gäbe es allerdings auch viele andere Kommunikationsformen wie E-Mails und Nachrichten, die über Messengerdienste versendet werden. Diese Kommunikationsformen würden sich im Hinblick auf den Schreibstil und in der Verwendung von den klassischen Medien unterscheiden. Es stellte sich die Frage, ob und wie Medien Schülerinnen und Schüler formen und wie sich die Wahrnehmung bei der Verwendung unterschiedlicher Medien verändert hat. Anschließend wurde das Wort an Frau Scharf weitergegeben, die sich mit der Frage befasste, woran Kinder Informationskompetenz fest machen. Hier zeige sich, dass sie eine Unterscheidung in wichtige und unwichtige Informationen vornehmen. Diese Fähigkeit Informationen zu gewichten, würde eher als allgemeine Kompetenz angesehen.

\section{Informationsverhalten und Informationskompetenz}

Sarah Stumpf und Bernhard Franke (Martin-Luther-Universität Halle-Wittenberg) thematisierten in ihrem Vortrag die Problematiken bei der Nachrichtendistribution in Social Online Networks. Dabei wurde deutlich, wie anfällig 
insbesondere Facebook für Verzerrungen ist. Social Media, Meinungsfreiheit und das Internet machten es möglich, dass jede Person Inhalte produzieren und konsumieren könne. Reichweite und Interaktion wären dabei Erfolgsfaktoren. In der Workshop-Phase widmeten sich die Teilnehmerinnen und Teilnehmer den Herausforderungen an den Betrieb von Social Networks im Zuge der Demokratisierung des Informationsflusses; wie Fachleute einen Beitrag dazu leisten, dass Falschmeldungen und Instrumentalisierungen gezielt erkannt werden, welche Aufgaben institutionelle Bildungsorganisationen haben um Informationskompetenz zu fördern und welche Fähigkeiten bei der Nutzung benötigt werden.

\section{Informationskompetenzvermittlung}

Stefan Dreisiebner (Universität Hildesheim) stellte in seinem Vortrag den Massiven Offenen Online Kurs (MOOC) ILO, »Information Literacy Online« vor. Dieser MOOC wird in unterschiedlichen Sprachen angeboten. Der MOOC ist Teil eines durch Erasmus+ geförderten Projektes, an dem mehrere Partneruniversitäten mitwirkten. Die Inhalte seien lizenzfrei veröffentlicht, damit sie individuell den Lehrzwecken angepasst werden können. Evaluierungsergebnisse ergaben, dass eine Einbindung in die Lehre zu einem Zuwachs an Fähigkeiten führe. Jedoch fällt der Zuwachs an Fähigkeiten und die Abschlussquote gering aus, wenn die Durchführung des MOOCs den Studierenden lediglich empfohlen wurde und nicht als aktiver Bestandteil in die Lehre integriert wurde.

Marcus Bölz (Fachhochschule des Mittelstandes) und Hans-Jakob Erchinger (Landesinstitut für schulische Qualitätsentwicklung) machten deutlich, dass journalistische Fähigkeiten ein wichtiger Bestandteil für die Vermittlung einer demokratischen Wertekultur seien. Bölz beschrieb, dass Medien sich im Zuge der Digitalisierung nicht substituieren, sondern differenzieren würden. Man synchronisiere sich permanent über digitale Medien. Doch auch andere Medien (bspw. Tageszeitung) erreichen immer noch Menschen und deren Inhalte seien nach wie vor relevant. Information sollte als offenes Gut gesehen und es sollte eine öffentlich-rechtliche Kommunikationsarchitektur geschaffen werden Ein Mittel Informationskompetenz zu befördern stelle der Journalismus dar, welcher das Potenzial hätte, Schülerinnen und Schüler mündig und neugierig zu machen, um die Gesellschaft zu erkunden, sich Konflikten zu stellen und zu diskutieren. Dafür würden Lehrkräfte als Multiplikatoren benötigt.

\section{Zukünftige Anforderungen}

Der letzte Tagungsvortrag von Inka Tappenbeck (Technische Hochschule Köln) und Antje Michel (Fachhochschule Potsdam) thematisierte zukünftige Anforderungen an Vermittlungspraktiken von Informationskompetenz. Die Informationsbedarfe verschiedener Gruppen seien unterschiedlich. Die Informationsobjekte würden sich je nach Fachrichtung unterscheiden, sodass Bibliotheken nicht für alle Gruppen notwendig seien. Dies führe zur Frage auf, ob es besser wäre, sich stärker auf einzelne Fachbereiche auszurichten und sich deren Arbeitsweise anzupassen. Beispiele hierfür seien einerseits die Literaturwissenschaften mit meist textlichen bzw. textbezogenen Inhalten und andererseits die Chemie, die Stoffe und deren Eigenschaften als Forschungsgegenstand betrachte.

Die Abschlusstagung des Projekts findet am 19. und 20. Juni 2020 in Hildesheim statt.

Deskriptoren: Tagung, Universität Hildesheim, Projekt, Informationskompetenz, Medien, Benutzerschulung

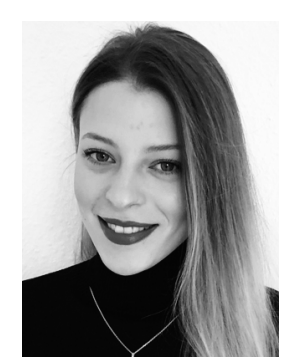

\author{
Daphné Çetta \\ Stiftung Universität Hildesheim \\ Institut für Informationswissenschaft und \\ Sprachtechnologie \\ Universitätsplatz 1 \\ 31141 Hildesheim \\ cetta@uni-hildesheim.de
}

Daphné Çetta ist seit 2019 wissenschaftliche Mitarbeiterin im Projekt Informationskompetenz und Demokratie an der Universität Hildesheim. Sie verfasste ihre Masterarbeit im Studiengang Internationales Informationsmanagement zum Thema „Gestaltungs- und Akzeptanzfaktoren von Videos für Lehramtsstudierende“.

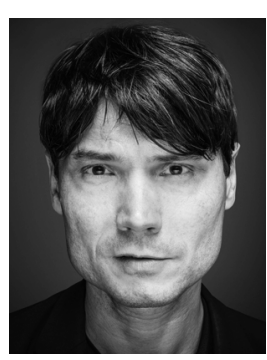

Prof. Dr. Joachim Griesbaum

Stiftung Universität Hildesheim

Institut für Informationswissenschaft und

Sprachtechnologie

Universitätsplatz 1

31141 Hildesheim

griesbau@uni-hildesheim.de

Joachim Griesbaum, seit 2001 wissenschaftlich im Bereich der Informationswissenschaft tätig, ist Professor für Informationswissenschaft an der Universität Hildesheim. Schwerpunkte in Lehre und Forschung sind Online Marketing, E-Learning und Informationsverhalten. 